JOURNAL DE PHYSIQUE

Colloque C2, supplément au $n^{\circ} 2$, Tome 46 , février 1985

page $\mathrm{C} 2-729$

\title{
EXPERIMENTAL POSSIBILITIES WITH UA2
}

\author{
G. Sauvage \\ Laboratoire de l'Accélérateur Linéaire, Université Paris-Sud, \\ 81405 Orsay, France
}

\begin{abstract}
Résumé - Nous donnons ici un bref rêsumé des résultats de physique marquants obtenus auprès du collisionneur Sp $\bar{p} S$ du CERN. L'accent est mis sur les Iimitations dues au fait que les faisceaux de protons et d'antiprotons ne sont pas polarisés.
\end{abstract}

Abstract - A brief summary of the most important physics results obtained with the Spps collider at CERN is given. The emphas is is put on the limitations due to the fact that both proton and antiproton beams are unpolarised.

The main results obtained in 1982-1983 by both experiments, UA1 and UA2, at the Sp p̈s collider at CERN, two-jet dominance and $W-Z$ discovery, correspond to an integrated luminosity of about $120 \mathrm{nb}^{-1}$. In the coming years, 1984 to 1986 , the energy of the beams will increase from $270 \mathrm{GeV}$ to $315 \mathrm{GeV}$ (the c.m. energy will increase from $\sqrt{S}=540 \mathrm{GeV}$ to $\sqrt{\mathrm{S}}=630 \mathrm{GeV}$ ) and the integrated luminosity will be multiplied by a factor 5 to 10 . The peak luminosity will be increased by an order of magnitude when the ACOL becomes available in 1987-1988. Despite the expected increase of the number of events in the coming years, there are limitations due to the fact that the beams are unpolarised, as we will see later.

Three topics will be considered (1) :

- jet physics and two-jet dominance

- intermediate vector bosons physics

- indications of new physics beyond the standard electro-weak model.

\section{JET PHYSICS AND TWO-JET DOMINANCE}

A striking feature of the hadronic physics at the CERN collider is the emergence of the two-jet dominance in agreement with QCD predictions. As soon as the total transverse energy observed in an event is high enough, about $100 \mathrm{GeV}$ in the case of UA2 (in a pseudorapidity interval $|\eta|<1$ ), the event is generally a two-jet event. The jet production properties, as the transverse momentum of the two-jet system, the structure functions of the proton (and antiproton), the two-jet angular distributions, and the jet fragmentation properties are in good agreement with QCD predictions and extrapolations from lower energy experiments. The two-jet production cross section is shown on the figure (UA2 results). The shaded area corresponds to different QCD predictions. The theoretical predictions are affected mainly by the following uncertainties : scale ambiguity (choice of $Q^{2}$ ) in the leading log. term, and related to it, the choice of the $\Lambda$ parameter in the strong coupling constant $\alpha_{S}\left(Q^{2}\right)$, as well as the parametrization used $f\left(\frac{\rho}{2} \mathrm{p}\right.$ and $\overline{\mathrm{p}}$ structure functions. A possible substructure of quarks and leptons (2) could manifest itself as a new contact interaction visible at large transverse momentum or at large mass and give deviations from pure QCD behaviour at large mass. This deviation is controlled by an energy scale $\xi$ parameter, characterizing the strength of this new interaction. The 1 imit put by UA2, $\xi>275 \mathrm{GeV}(95 \% \mathrm{CL})$ is dominated by the theoretical uncertainties in pure QCD calculation $(\xi=\infty)$. It has been pointed out $2 \xi$ at this conference that the theoretical uncertainties are much lower when one considers cross section differences with polarized proton beams in the CERN collider. This is a way to improve the 1 imit on the $\xi$ parameter. 


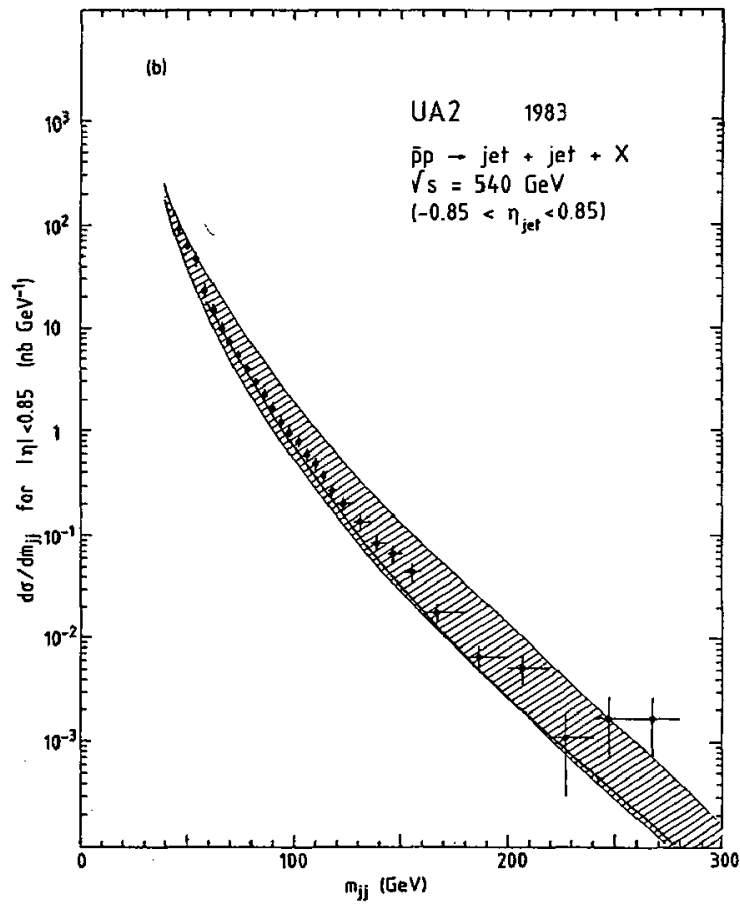

INTERMEDIATE VECTOR BOSON PHYSICS

- W, Z discovery

The $\mathrm{W}$ and $\mathrm{Z}$ vector bosons have been discovered through their leptonic decays :

$$
\begin{aligned}
& W^{ \pm} \rightarrow \ell^{ \pm} \varphi \\
& Z^{0} \rightarrow \ell^{+} \ell^{-}
\end{aligned} \quad \text { where } \ell \quad \text { is either an electron or a muon }
$$

In these decays the lepton $\ell$ has a high transverse momentum, of the order of half the mass of the vector boson ( $\sim 25$ to $40 \mathrm{GeV} / \mathrm{c}$ ) and the background conditions due to hadronic processes are favourable. The next table summarises the number of events collected in both UAl and UA2 experiments.

$\begin{array}{ccccc} & W \rightarrow \text { ev } & W \rightarrow \mu \nu & z^{\circ} \rightarrow \mathrm{e}^{+} \mathrm{e}^{-} & \mathrm{z}^{\circ} \rightarrow \mu^{+} \mu^{-} \\ \text {UA1 } & 52 & 14 & 4 & 5 \\ \text { UA2 } & 37 & - & 8 & -\end{array}$

(UA2 has no muon detection).

The mass of these particles have been determined :

$$
\begin{aligned}
& \mathrm{m}_{\mathrm{w}}=80.9 \pm 1.5 \pm 2.5 \mathrm{GeV} / \mathrm{c}^{2}(\mathrm{UA1}) \mathrm{m}_{\mathrm{W}}=83.1 \pm 1.9 \pm 1.3 \mathrm{GeV} / \mathrm{c}^{2} \text { (UA2) } \\
& \mathrm{m}_{\mathrm{Z}}=95.6 \pm 1.4 \pm 2.9 \mathrm{GeV} / \mathrm{c}^{2} \text { (UA1) } \mathrm{m}_{\mathrm{Z}}=92.7 \pm 1.7 \pm 1.4 \mathrm{GeV} / \mathrm{c}^{2} \text { (UA2) }
\end{aligned}
$$

and are in good agreement with the predictions of the SU(2) $x \quad U(1)$ standard model. The production cross sections are also in agreement with the standard model.

Due to the $\mathrm{V}-\mathrm{A}$ nature of the coupling of the $\mathrm{W}$ to both the $q \overline{\mathrm{q}}$ and $\ell^{ \pm} \gamma$ systems, the angular distribution of the decay lepton $l^{-}$is asymetric and has the form : $\mathrm{dN}\left(e^{ \pm}\right) / \mathrm{d} \cos \theta^{*}=\left(1 \pm \cos \theta^{*}\right)^{2}$

Both experiments have measured this asymmetry. UA1 has determined the mean value of $\cos \theta^{*}$ and found : $\left\langle\cos \theta^{*}\right\rangle=0.4 \pm 0.1$ in agreement with the expected 
value $\left\langle\cos \theta^{*}\right\rangle=0.5$. UA2 has determined the value of the ratio $A / V$ of the axial vector coupling to the vector coupling and found :

$$
|\mathrm{A} / \mathrm{V}|=1.0+0.5
$$

The error bars will, of course, diminish with increased statistics in the coming years. But it is important to note that, with unpolarised beams, the angular distribution of the decay lepton is not sensitive to the sign of the axial vector to vector ratio of the coupling. In other words one cannot distinguish, with such measurements, between a left handed $W$ (as predicted by the standard mode1) and a right handed $W$. The use of polarised protons in the spps collider gives rise to asymmetries sensitive to the sign of the coupling. A possible way out to determine this sign, is to measure the polarisation of the final lepton, e.g., by studying the decay channel $W \rightarrow \tau \nu$ and the decay products of the $\tau$. However the background conditions are more severe as one is dealing with leptons ( $\mu$ or e) of smaller transverse momenta.

The previous remark about the $W$ applies also to any new $W$ of higher mass, if it exists. In such a case, the determination of the $V-A$ or $V+A$ nature of the coupling will be of primary importance. The use of ${ }^{2}$ polarised proton beam in the sp $\bar{p}$ collider gives rise also to spin asymmetries (3) in the case of the $z^{\circ}$ production well predicted in the framework of the standard electroweak model.

\section{- top quark search}

The sixth quark, the top quark, not seen in $\mathrm{e}^{+} \mathrm{e}^{-}$annihilations because of its high mass, can be produced at the CERN collider and studied through the following decay chain : $p \vec{p} \rightarrow W+\ldots, W \rightarrow t \bar{b}, \quad t \rightarrow b$ e $v$ The topology of such an event is the presence of a lepton with a moderate transverse momentum $(\sim 10-15 \mathrm{GeV} / \mathrm{c})$, of 2 jets corresponding to the $\bar{b}$ and $b$ quarks and of missing transverse energy corresponding to the unseen neutrino. UA1(4) has recently reported to have isolated six such events ( 3 electrons and 3 muons) compatible with the above assumption, and derived limits on the mass of the $t$ quark: $30 \mathrm{GeV} / \mathrm{c}^{2}<\mathrm{m}_{\mathrm{t}}<50 \mathrm{GeV} / \mathrm{c}^{2}$. The use of a polarised beam in the CERN collider may also help to isolate the signal as it gives rise to asymmetries in the semileptonic decay of the top.

$$
\text { - hadronic decay of the } W, Z: W, Z \rightarrow q \bar{q} \quad(q=u, d, s, \ldots)
$$

The main difficulty encountered to detect these decay channels, is the abundant two-jet QCD production. The hadronic $W, Z$ decay will appear as a mass bump in the two-jet mass distribution. For example, UA2 sees an effect of only 1 to 1.5 standard deviation : $\mathrm{N}_{\mathrm{W}}=108 \pm 70$ for an expected number of hadronic $W$ decays of $\mathrm{N}_{W}=150$. In the coming years, the increased number of events will improve somewhat the statistical significance of such an effect. However, there are two ways of improving substantially the situation. One can improve the mass resolution by using better calorimeters to measure the energy of the jets. One can also use a polarised proton beam to separate the parity non conserving 2 jet production through the $w$ channel from the parity conserving 2 jet QCD production.

\section{BEYOND THE STANDARD ELECTROWEAK MODEL}

A small number of unexpected events have been observed by UAl and" UA2 which could indicate the existence of a new physics beyond the standard electroweak model. Here are surmarized the different kinds of events.

$$
z^{0} \rightarrow \ell^{+} \ell^{-} \gamma \quad .3 \text { radiative } z^{\circ} \text { decays have been observed, } 2 \text { in UA1 and } 1 \text { in }
$$

UA2. These events may be produced by internal bremsstrahlung although their probability is small. For example, the probability of observing such a radiative event in the UA2 sample $\left(8 \mathrm{Z}^{\circ}\right.$ ) is less than $4 \%$. The corresponding radiative $W$ decay, $W \rightarrow \ell v Y$ has not been observed despite the higher number of $W_{S}^{\prime}$. 
- mass bump in the two-jet mass distribution. Looking for a mass bump beyond the $W, Z$ mass a three standard deviation effect has been observed by UA2, at a mass of about $150 \mathrm{GeV} / \mathrm{c}^{2}$.

- e"v" jets events. UA2 has isolated three events where a high transverse momentum electron is produced in association with a high missing transverse energy (assumed to be due to an unseen neutrino) and with hard jets. Assuming that the ev system has the mass of the $W$, the mass of the ev jets system cluster at a mass of about $170 \mathrm{GeV} / \mathrm{c}^{2}$. The significance of this observation is weakened by the fact that this mass region is kinematically favoured by the selection criteria.

- single photon and single jet events. 2 single photon and 5 single jet events associated with a high missing transverse energy have been observed by the UA1 experiment. These events, or their rates, are not explained by conventional sources. Assuming that they are the results of the decay of a high mass state $X$ decaying in $z^{\circ}+$ jet followed by a decay of a $z^{\circ}$ into two unseen neutrinos, the allowed window for the $X$ mass is around $170 \mathrm{GeV} / \mathrm{c}^{2}$. However, if such a state exists, the $\mathrm{Z}^{\circ}$ decay into two-jets should have been observed and there is no sign of a mass bump in the two-jet or three-jet mass distribution in UAl data.

For all above quoted results, there is a clear need for more statistics. The coming years will show which of these effects were due to statistical fluctuations and which are genuine, For the interpretation of this new phenomena, the use of a polarised proton beam in the CERN collider can be important,

As a conclusion the interest of using polarised proton beams in the CERN Collider has been seen to be twofold :

- it may help to improve the background conditions, when, for example one is studying a parity non conserving effect, as QCD background is parity conserving.

- it may bring new information very difficult to obtain with unpolarised beams, even with an improved statistics.

However, the low cross sections of interesting phenomena to be studied, do not allow to lose too much on the luminosity of the collider when one uses polarised proton beams.

\section{REFERENCES}

(1) - Recent review of UA1 and UA2 results can be found in : RHOLF J., Plenary Report (UAl) results REPELLIN J.P., Plenary Report (UA2) Results at the 22nd International Conference on High Energy Physics, Leipzig, DDR, 19 th-25th July, 1984.

(2) - RÜCKL R., Spin effects from quark and lepton substructure for future machines (this proceeding $p$. )

(3) - For example : KINNUNEN R., LINDFORS J., Nucl. Phys. B189 (1981) 63-86 CHIAPPETTA P., SOFFER J., Spin asymmetries for $W$ and $Z$ production in $p \bar{p}$ collisions, CPT-84/p.1650 (to be published)

(4) - ARNISON G. et a1., Associated production of an isolated large-transverse momentum lepton (electron or muon) and two-jets at the CERN PP collider, UAl Collaboration CERN-EP/84-134. 